\title{
Attractive electron mobility in (113) n-type phosphorus-doped homoepitaxial diamond
}

\author{
Marie-Amandine PINAULT-THAURY ${ }^{1}$, Ingrid STENGER, Rémi GILLET, Solange \\ TEMGOUA, Ekaterina CHIKOIDZE, Yves DUMONT, François JOMARD, Thierry \\ KOCINIEWSKI, and Julien BARJON
}

Université Paris-Saclay, UVSQ, CNRS, GEMaC, 78000 Versailles, France

https://doi.org/10.1016/j.carbon.2021.01.011

\begin{abstract}
s
A (113) diamond homoepilayer doped with phosphorus is grown. It presents high crystalline quality and n-type conductivity with a maximum electron mobility of $355 \mathrm{~cm}^{2} / \mathrm{V} . \mathrm{s}$ at $450 \mathrm{~K}$. Its electrical properties are compared to those measured on conventionally oriented (100) and (111) homoepilayers synthetized in the same reactor with similar phosphorus content ( 1$2 \times 10^{18} / \mathrm{cm}^{3}$ ). The (113) layer presents higher electron mobility than the (100) film, despite a comparable compensation ratio. Besides, above $450 \mathrm{~K}$ the (113) electron mobility is also higher than the one of the low compensated (111) sample. This shows the attractive character of the (113) diamond orientation for n-type doping.
\end{abstract}

${ }^{1}$ Corresponding author. Tel: 0033-139-254-489. E-mail: marie-amandine.pinault-thaury@uvsq.fr 


\section{Introduction}

With tremendous physical properties, diamond is considered to be the ultimate semiconductor for high power electronics [1]. Indeed, diamond is awaited to endure high electric fields with low leakage current. It is then a candidate of choice for high voltage and high temperature power electronics. Compared to other wide bandgap semiconductors (ex: $\mathrm{ZnO}$ and $\mathrm{GaN}$ ), high mobility of the holes is a prime advantage for bipolar devices. However, diamond presents a doping asymmetry (as $\mathrm{ZnO}$ for example). While its p-type doping with boron in substitution for carbon is easy [2] [3] [4], its n-type conductivity is difficult to achieve. Being a major issue for the fabrication of diamond-based bipolar devices, $n$-type doping is an active research topic [5] [6] [7].

Even if some incursions were made on the (110) orientation [8], the conventional crystalline orientations used for diamond are (111), that especially suffers from easy crystalline defect formation, and (100), on which better electronic properties are generally observed on larger surfaces [9]. Lying in between (111) and (100) [10], (113) is a stable growth orientation during chemical vapor deposition (CVD) [11] and could allow obtaining enlarged crystals [12]. The high potential of the (113) orientation was revealed recently thanks to the works of Lesik et al. [10] and Tallaire et al. [13]. For achieving a given film thickness together with a dedicated doping level, a wide growth window is a precious advantage. Tallaire et al. [13] show that the use of (113) orientation substrates for p-type boron doped diamond homoepitaxy allows extension of the optimal MPCVD growth window, compared to conventional (100) orientation (ex: higher growth temperatures and growth rates). Moreover, the (113) p-type doping has been reached with homoepilayers presenting high crystalline quality whatever the layer thicknesses, varying from a few hundred of micrometers to free-standing plates. No unepitaxial defects (as crystallites) were observed unlike on (100) orientation. A freestanding plate of (113) heavily boron doped CVD diamond exhibits low resistivity compared to (100) orientation. Even if heavily boron doped diamond is reached on (111) orientation substrates, the formation of twins and growth defects forbidden the growth of thick high boron doped (111) layers. Thus, compare to (100) and (111) orientations, it is possible to consider highly conductive (113) substrates that are required, for example, in vertical Schottky diodes. By improving boron doping efficiency in high-quality thick diamond, (113) orientation could represent a good tradeoff between (100) and (111) orientations. As a result, the use of (113) diamond seems very attractive for power electronic devices as bipolar devices.

With an ionization energy of $0.6 \mathrm{eV}$ [14] phosphorus is the donor that gives the highest n-type conductivities in diamond [15] [16] [17] [18]. While phosphorus atoms get incorporated in substitutional sites in (111) homoepilayers rather easily [19], the growth of (100) n-type diamond with phosphorus is quite difficult and requires a specific and narrow window of growth parameters [9] [20]. In our previous work [21], we found a window of growth parameters that results in high crystalline quality (113) epilayers with full incorporation of phosphorus donors in carbon sites [21]. The (113) phosphorus incorporation efficiency and the maximum phosphorus content lies between the reported values for (100) and (111) [20] [22] [23].

Diamond use deep energy level carriers. Thus, it has increased numbers of carriers at high temperature. Donato et al. [24] recently highlights the high operating temperature of $450 \mathrm{~K}$ by compare diamond to $4 \mathrm{H}-\mathrm{SiC}$ and $\mathrm{GaN}$. Even if diamond is superior at $400 \mathrm{~K}$, the gap in the performance is significantly amplified above $450 \mathrm{~K}$ where many physical parameters are strongly modified (carrier activation, carrier mobility, density of states, etc). Hence, compare to $4 \mathrm{H}-\mathrm{SiC}$ and $\mathrm{GaN}$, diamond devices might present higher current density (33\% and $20 \%$, respectively), less losses (28\% and 19\%, respectively) and heatsink volume. As a result, new diamond power devices might find their place in the future electronics market, especially for high temperature applications, starting from $450 \mathrm{~K}$ (e.g. more electric aircraft). 
Here, we report on the electrical properties of phosphorus-doped diamond grown on the (113) orientation. The results are confronted to those obtained on state-of-the-art (111) and (100) films containing similar phosphorus concentration. All three homoepilayers were synthetized in our MPCVD reactor. We show that our growth conditions give rise to (113) ntype layer presenting higher conductivities than a (100) film, and open new perspectives for the n-type doping of diamond and more generally for bi-polar devices.

\section{Experiments and results}

The phosphorus-doped homoepitaxial layers have been grown on type Ib high pressure high temperature (HPHT) diamond substrates. The (111) and (100) substrates present $2 \times 2 \mathrm{~mm}^{2}$ and $3 \times 3 \mathrm{~mm}^{2}$ square surfaces with a thickness of $0.5 \mathrm{~mm}$ and $1.5 \mathrm{~mm}$, respectively. In contrast the (113) plates have a cylindrical shape (see the optical picture in the inset of Figure 1.a) with a diameter of $2 \mathrm{~mm}$ and a thickness of $0.5 \mathrm{~mm}$ [10]. The miscut angle of each substrate has been measured by $\mathrm{X}$ ray diffraction. It is of $2.5^{\circ}$ and $1.5^{\circ}$ along random directions for (111) and (113) plates, and of $3.5^{\circ}$ along [110] directions for the (100) sample. Before introduction of the substrates into the growth reactor, organic and metallic contaminations were removed with a chemical treatment [17].

Samples were fabricated in our laboratory-built reactor allowing microwave plasma chemical vapor deposition (MPCVD) associated with the metal-organic technology for doping using a liquid phosphorus precursor [25]. As a dopant precursor we used tertiarybutylphosphine (TBP: $\mathrm{C}_{4} \mathrm{H}_{11} \mathrm{P}$ ) vapor diluted in hydrogen. The substrate temperature was measured with a dualwavelength Williamson pyrometer (the relative uncertainty is 10\%). Growth parameters specific to each surface orientation have been used to achieve high crystalline quality and an almost complete incorporation of phosphorus in donor sites as shown in our previous works [9] [16] [21] [26]. Considering the notations [P] for the phosphorus atomic content and $\mathrm{N}_{\mathrm{D}}$ for the phosphorus donor concentration, our layers present $[P] \approx N_{D}$. For the (111) [23] and (100) [9] surfaces, the n-type doping was carried out with TBP giving phosphorus concentration in the gas phase at a level of $[\mathrm{P}] /[\mathrm{C}]_{\text {gas }}=0.01 \%$ and $1.2 \%$, respectively. In contrast, the $n$-type doping of the (113) layers was non-intentionally doping (doping due to residual phosphorus in the vacuum chamber), as shown in our previous report [21]. The samples are labeled A, B, C with respect to the (100), (113) and (111) orientations. The growth parameters and the main characteristics of the samples are summarized in Table 1.

Secondary ion mass spectrometry (SIMS) was used to measure the phosphorus content in the samples (the relative uncertainty is $\sim 10 \%$ ) as described elsewhere [17] [21]. The phosphorus content in the three epilayers is homogenous in-depth, as attested by the almost flat SIMS profiles from the surface to the epilayer interface with the HPHT substrate. As an example Figure 1.a shows the phosphorus concentration profile of sample B made on (113) orientation. The three samples present comparable phosphorus concentration around $1-2 \times 10^{18}$ $\mathrm{at} / \mathrm{cm}^{3}$. For the (113) sample, this value is in between our previous reported values, $7 \times 10^{16}$ and $3 \times 10^{19} \mathrm{at} / \mathrm{cm}^{3}$, for non-intentionally doped (113) homoepilayers grown with similar MPCVD conditions [13]. The phosphorus atoms incorporated in the non-intentionally doped layers are not coming from the gas line but from the reactor itself. More precisely some internal parts of the vacuum chamber are suspected to outgassing phosphorus from their surface thanks to the heat of plasma radiations. The released phosphorus atoms are then coming into the gas mixture and participate to the growth. The internal parts of the vacuum chamber are polluted with phosphorus because of the use of various and high TBP overpressure needed for other type of growth studies [27]. Even if it is time consuming and expensive, the control and decrease of the phosphorus concentration in (113) orientation is possible thanks to clean-up and/or replacement of critical internal parts of the vacuum chamber. Once done, at first the use of TBP low overpressure should be favored to reach low phosphorus concentrations in (113) orientation. 


\begin{tabular}{|l|l|l|l|}
\hline \multicolumn{1}{|c|}{ Sample } & A & B & C \\
\hline Orientation & $(100)$ & $(113)$ & $(111)$ \\
\hline Miscut angle & $3.5^{\circ}$ & $1.5^{\circ}$ & $2.5^{\circ}$ \\
\hline Pressure $($ mbar) & 75 & 50 & 50 \\
\hline Growth temperature $\left({ }^{\circ} \mathrm{C}\right)$ & 1130 & 900 & 890 \\
\hline Total gas flow $(\mathrm{sccm})$ & 400 & 1000 & 1000 \\
\hline$[\mathrm{C}] /\left[\mathrm{H}_{2}\right]_{\text {gas }}(\%)$ & 1.80 & 0.05 & 0.05 \\
\hline$[\mathrm{P}] /[\mathrm{C}]_{\text {gas }}(\%)$ & 1.20 & 0 & 0.01 \\
\hline Duration & $3 \mathrm{~h}$ & $6 \mathrm{~h}$ & $6 \mathrm{~h}$ \\
\hline Growth rate $(\mu \mathrm{m} / \mathrm{h})$ & 5.27 & 0.16 & 0.20 \\
\hline$[\mathrm{P}]\left(\right.$ at $\left./ \mathrm{cm}^{3}\right)$ & $1.0 \times 10^{18}$ & $2.0 \times 10^{18}$ & $1.7 \times 10^{18}$ \\
\hline Compensation ratio, $\mathrm{k}$ & $83 \%$ & $80 \%$ & $10 \%$ \\
\hline Activation energy, $\mathrm{E}_{\mathrm{A}}(\mathrm{eV})$ & 0.56 & 0.55 & 0.59 \\
\hline Electron mobility, $\mu_{\mathrm{n}}\left(\mathrm{cm}^{2} / \mathrm{V} . \mathrm{s}\right) @ 450 \mathrm{~K}$ & 111 & 355 & 217 \\
\hline
\end{tabular}

Table 1: Parameters for the growth of homoepitaxial layers on (100), (113) and (111) diamond substrates and physical properties extracted from the SIMS and electrical measurements analysis.

The optical quality has been estimated using cathodoluminescence (CL) spectroscopy on an equipment optimized to analyze the 200-1000 nm range emission [28]. The samples are cooled down in a JEOL7001F scanning electron microscope (SEM) close to the liquid helium temperature. The CL spectra were obtained using a $10 \mathrm{keV}$ electron beam which corresponds to approximately $0.9 \mu \mathrm{m}$ of penetration depth of electrons. For all the samples, the CL spectra exhibit a dominant peak corresponding to the neutral phosphorus bound exciton recombination $\mathrm{P}^{0} \mathrm{X}_{\mathrm{TO}}$ at $5.19 \mathrm{eV}$ when assisted by the emission of a transverse optical (TO) phonon [29]. Figure 1.b shows the CL spectrum of sample B made on (113) orientation. The no-phonon phosphorusbound exciton recombination $\mathrm{P}^{0} \mathrm{X}_{\mathrm{NP}}$ is also observed at $5.33 \mathrm{eV}$. The linewidths of the $\mathrm{P}^{0} \mathrm{X}_{\mathrm{TO}}$ and $\mathrm{P}^{0} \mathrm{X}_{\mathrm{NP}}$ line are around $10.5 \mathrm{meV}$ and $2.2 \mathrm{meV}$ indicating that the crystallinity of the epilayers is similar to good quality (111) phosphorus doped epilayers [30].

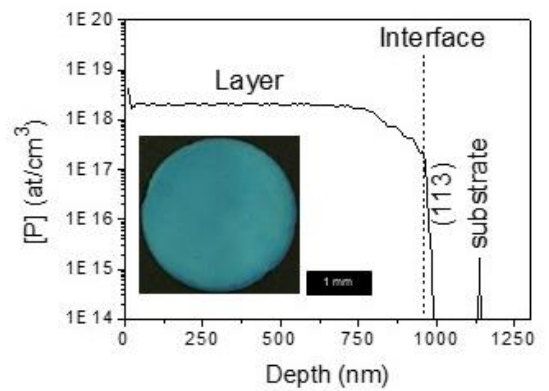

(a)

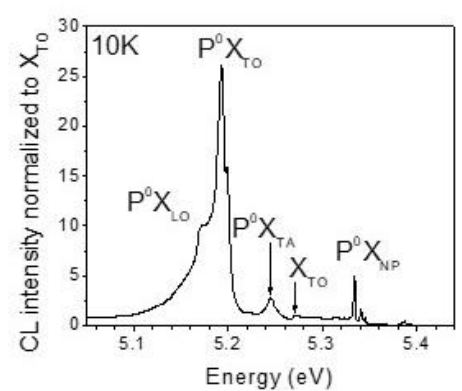

(b)

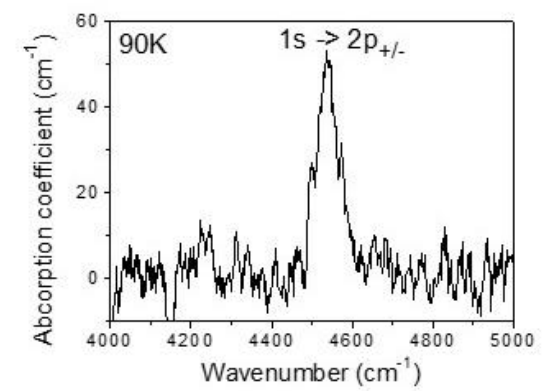

(c)

Figure 1: Physical characteristics of phosphorus-doped epilayer grown on a (113) substrate (sample B): (a) SIMS profile of the phosphorus concentration) (inset: optical image of the sample surface); (b) CL spectrum attesting of the incorporation of phosphorus in donor sites through the bound exciton $\mathrm{P}^{\circ} \mathrm{X}_{\mathrm{TO}}$; (c) FTIR absorption spectrum showing the presence of uncompensated phosphorus donors, through the observation of the $1 \mathrm{~s} \rightarrow 2 \mathrm{p}_{+/-}$electronic transition from neutral phosphorus donors. 
The presence of uncompensated phosphorus donors in our phosphorus-doped samples has been attested by infrared (IR) absorption in a transmission geometry. The experiment was performed at $90 \mathrm{~K}$ in a BOMEM DA8 Fourier-transform interferometer (FTIR), from 1800 to $8500 \mathrm{~cm}^{-1}$ using an InSb detector and a spectral resolution of $2 \mathrm{~cm}^{-1}$. For the three samples, we observe the absorption line at $4540 \mathrm{~cm}^{-1}$ corresponding to the $1 \mathrm{~s} \rightarrow 2 \mathrm{p}_{+/-}$electronic transition of neutral phosphorus [31]. Figure 1.c shows the FTIR spectrum of sample B made on (113) orientation. Since the absorption coefficient is proportional [31] to the neutral phosphorus donor concentration $\mathrm{N}_{\mathrm{D}}^{0}=\mathrm{N}_{\mathrm{D}}-\mathrm{N}_{\mathrm{A}}$, (where $\mathrm{N}_{\mathrm{A}}$ is the concentration of compensating acceptors) the absorption experiments evidence that the studied epilayers present a significant amount of uncompensated phosphorus donors. As a result, a n-type electrical conductivity is expected for phosphorus-doped diamond grown on the three surface orientations.

The DC electrical measurements were performed by resistivity and Hall effect in a Van der Pauw configuration from $\sim 300 \mathrm{~K}$ to $850 \mathrm{~K}$ under a DC magnetic field of $1.6 \mathrm{~T}$, on a high impedance laboratory developed setup. Prior to the electrical measurements, the as-grown diamond film surfaces were chemically oxidized in order to remove the surface conductive layer due to the hydrogen termination [25]. For electrical contacts on the (100) epilayer (sample A), our surface microstructuration process [27] was applied before deposition of metallic contacts (Ti/Au) by sputtering. For epilayers grown on (113) and (111) orientations (samples B and $\mathrm{C}$ ), the electrical contacts were made with $\mathrm{Ti} / \mathrm{Pt} / \mathrm{Au}$ electrods. All contacts have been annealed at $600^{\circ} \mathrm{C}$ for 30 min under $\mathrm{N}_{2}$ atmosphere and present a sufficiently low rectifying characteristic to ensure reliable data from electrical measurements. A negative Hall voltage was measured over the whole $300-850 \mathrm{~K}$ temperature range, showing the conductivity is driven by electrons (n-type) in our samples.

Figure 2.a shows the temperature dependence of the resistivity, $\rho$ (lozenges), and the carrier concentration, $\mathrm{n}$ (circles), for the 3 samples, A, B and C, grown on (100), (113) and (111) orientations, respectively. Free electrons are thermally activated, as a result of phosphorus donor ionization. From resistivity versus 1000/T dependence, it is clear that band conduction is the dominant conduction process observed for the three surface orientations over the temperature range. It leads to an almost exponential decrease of the layer resistivity with temperature (and the related increase of the carrier concentration: see fitted curves in Fig.2(a)). In the low temperature region, a weak deviation is observed, due to variable range hopping [32]. The (100) sample presents the highest resistivity. At room temperature (RT), the (113) and (111) epilayers present a similar resistivity in the $10^{5} \Omega . \mathrm{cm}$ range which is more than 10 times lower than the one of the (100) layer.

From the temperature dependence of the carrier concentration, we deduce independently the impurity-to-band activation energy $E_{A}$ and the compensation ratio $k=N_{A} / N_{D}$. See ref [33] for more details on the fitting procedure. The extracted values are reported in Table 1 and the fitted curves are plotted (lines) in Fig. 2.a. The (111) sample presents a much lower compensation ratio of $8 \%$ together with an activation energy of $0.59 \mathrm{eV}$ as usually reported previously for n-type (111) [34]. The (100) film shows a high compensation ratio of $86 \%$ with a lower activation energy of $0.56 \mathrm{eV}$, in accordance with previous reports [35] [36]. In the case of (113) epilayer, the compensation ratio and the activation energy are close to the one of the (100) sample: $80 \%$ and $0.55 \mathrm{eV}$, respectively. The SIMS analysis reveals boron contamination in the (111) film at the level of $2 \times 10^{16} \mathrm{at} / \mathrm{cm}^{3}$ while no boron is detected in the (113) and (100) samples (even by CL spectroscopy: [B] $<10^{14} \mathrm{at} / \mathrm{cm}^{3}$ ). This suggests that the compensating acceptors are mainly coming from other defects than boron (ex: complexes involving carbon vacancies or hydrogen impurities) [37]. The identification of compensating centers is still an open issue for improving the conductivity of n-type diamond and remains under investigation. However, one can explain their origins. Indeed, diamond epitaxy results from a competition 
between diamond etching and diamond growth thanks to the high level of hydrogen contained in the gas mixture (more than 90\%). When the HPHT Ib substrate is exposed to the plasma, its top surface is etched only in the first stage of the layer epitaxy while its edges are longer etched before being covered by diamond layer over the growth process. Impurities coming from the substrate (as nitrogen) are then released in the gas mixture. Moreover, incorporation of other impurities into the gas mixture might also come from the internal parts of the vacuum chamber due to outgassing with plasma radiation heating (as boron). All those contaminating impurities in the gas mixture participate to the growth process. Structural defects (as dislocations) might appear also during the epitaxial growth. Without being directly and easily observable, those defects can act as compensators by themselves (boron, dislocations, etc.) or by complexing with others $(\mathrm{N}-\mathrm{V}, \mathrm{P}-\mathrm{V}, \mathrm{P}-\mathrm{H}, \mathrm{V}-\mathrm{H}$, etc.). Thus, the origin of compensators can come from contaminations from the substrate and the internal parts of the reactors but also from structural defects induced by the growth itself.

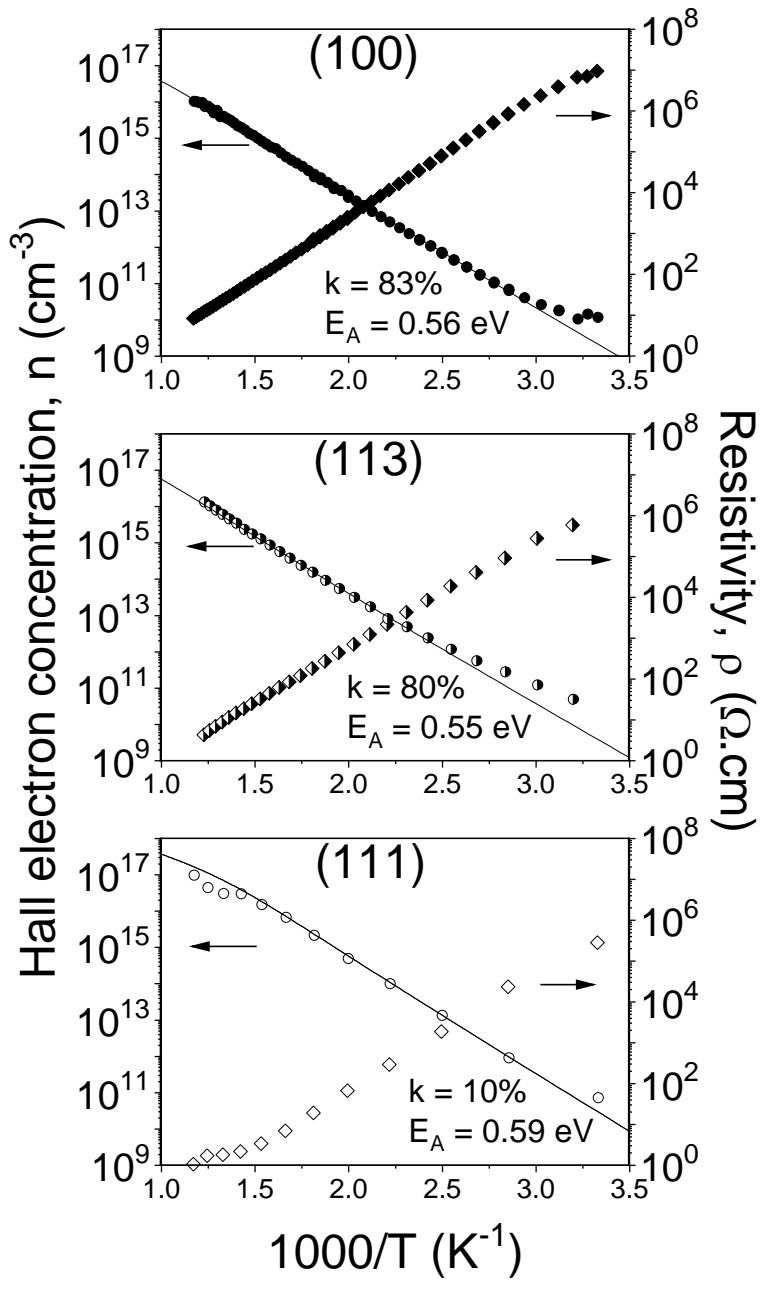

(a)

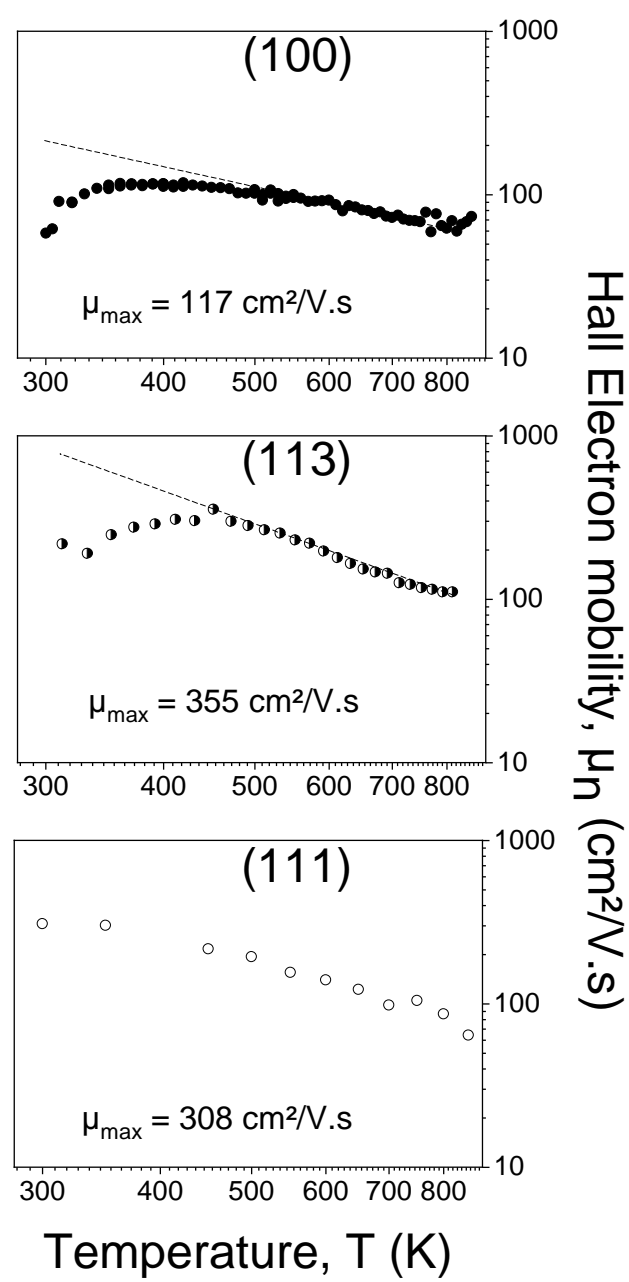

(b)

Figure 2: Electrical properties of the homoepitaxial diamond layers grown on (100), (113) and (111) surfaces having the same phosphorus concentration $[\mathrm{P}]=1-2 \times 10^{18} \mathrm{at} / \mathrm{cm}^{3}$ : temperature dependences of (a) electron concentration, $\mathrm{n}$ (circles, left axis), and layer resistivity, $\rho$ (lozenges, right axis); (b) electron mobility, $\mu_{\mathrm{n}}$ (in $\log -\log$ scale). For $\log \left(\mu_{\mathrm{n}}\right)$ versus $\log (\mathrm{T})$, the dashed lines of the (100) and (113) samples are extrapolations of the lattice scatterings high-T behavior. 
While diamond-based devices are supposed to work at high temperature $\left(>150^{\circ} \mathrm{C}\right)$, it is worth to evaluate the temperature dependence of the electron mobility, $\mu_{\mathrm{n}}$, (reported in Figure 2.b) for each diamond sample. For the (111) orientation, $\mu_{n}$ presents a maximum value of 308 $\mathrm{cm}^{2} / \mathrm{V}$.s at RT and then decreases with increasing temperature. (100) and (113) samples present increasing $\mu_{\mathrm{n}}$ up to $\sim 450 \mathrm{~K}$, with maximum values, respectively of 117 and $355 \mathrm{~cm}^{2} / \mathrm{V} . \mathrm{s}$., followed by a more or less significant decrease. Whatever the temperature, $\mu_{\mathrm{n}}$ of (113) sample always remains higher than $100 \mathrm{~cm}^{2} /$ Vs. unlike (111) and (100) samples. From $\log \left(\mu_{\mathrm{n}}\right)$ versus $\log (\mathrm{T})$ dependence, one can distinguish (i) the high-T behavior mostly due to lattice scatterings (acoustic phonons, optical phonons, intervalley phonons) with $\mathrm{T}^{-\beta}$ dependence where $\beta$ ranges from 2 to 0 and a value of $3 / 2$ for pure acoustic phonons scattering [38], and (ii) the lower-T behavior $\mathrm{T}^{\alpha}$ associated all sources of defects (ionized or neutral impurities). Indeed, according to Pernot et al. [38] on such temperature range, the factor limiting the electron mobility in (111) diamond epilayers is mainly intrinsic scattering due to acoustic phonons, thanks to the relatively weak compensation of the (111) layers. In our case, we found values of high-T $(\mathrm{T}>450 \mathrm{~K})$ lattice contribution $\beta$ coefficient of $0.9,1.8$ and 2 for (100), (111) and (113) respective orientations. From low-T behavior (300K-450K), electron mobility of (100) and (113) samples is mostly limited by the ionized impurities, probably associated residual acceptors involved in high compensation levels. Despite the fact that (113) film is highly compensated, it is remarkable that its electron mobility stays at higher values than the one reached on (100) layer over the whole temperature range and on (111) layer above 450K. Furthermore, if we extrapolate the high-T dependence of $\log \left(\mu_{\mathrm{n}}\right)$ versus $\log (\mathrm{T})$ to lower temperatures, associated mostly to lattice scatterings (see dashed lines in figure 2.b), we can expect even higher electron mobility on the (113) orientation, for less defective diamond (less ionized and/or neutral impurities). Then, improving the growth conditions or finding ex-situ treatment to reduce compensation in (113) phosphorus-doped diamond might allow higher electron mobilities, even higher than $1000 \mathrm{~cm}^{2} / \mathrm{V} . \mathrm{s}$. , the one predicted for (111) [39].

\section{Conclusion}

In this work we show that the growth window found for (113) phosphorus-doped diamond allows the synthesis of n-type conductive layer. Even with high compensation ratio the (113) CVD layer presents better electrical properties than on (100) orientation, currently preferred for electronics. Such results show that there is room for improvement on the (113) orientation. Indeed understanding the limitations of electron mobility in n-type diamond will be of prime importance to develop diamond electronics. Our results are quite attractive for the realization of future (113) diamond bipolar devices.

\section{Acknowledgements}

The authors would like to thank C. MER-CALFATI and B. BERINI for technical support on metal deposition; J. LABBÉ, Y. OUALI, N. TEMAHUKI for realization of the Hall effect measurements; and the French National Research Agency (ANR) for funding this work under the project MOVeToDiam N ${ }^{\circ}$ ANR-17-CE05-0019-02 and the CNRS under the Tremplin 2019 grant.

\section{References}

[1] S. Shikata "Single crystal diamond wafers for high power electronics" Diamond and Related Materials 65, 168-175 (2016), https://doi.org/10.1016/j.diamond.2016.03.013

[2] S.A. Bogdanov, A.L. Vikharev, M.N. Drozdov, D.B. Radishev, S.A. Bogdanov, A.L. Vikharev et al. "Synthesis of thick and high-quality homoepitaxial diamond with high boron doping level: oxygen effect" Diamond and Related Materials 74, 59-64 (2017), https://doi.org/10.1016/j.diamond.2017.02.004 
[3] S. Ohmagari, H. Yamada, H. Umezawa, N. Tsubouchi, A. Chayahara, Y. Mokuno "Growth and characterization of freestanding $\mathrm{p}+$ diamond (100) substrates prepared by hot-filament chemical vapor deposition" Diamond and Related Materials 81, 33-37 (2018), https://doi.org/10.1016/j.diamond.2017.11.003

[4] J. Achard, R. Issaoui, A. Tallaire, F. Silva, J. Barjon, F. Jomard et al. "Freestanding CVD boron doped diamond single crystals: A substrate for vertical power electronic devices?" Physica Status Solidi (a) 209 (9), 1651-1658 (2012), https://doi.org/10.1002/pssa.201200045

[5] N. Roschewsky, T. Matsumura, S. Cheema, F. Hellman, T. Kato, S. Iwata et al. "Spinorbit torques in ferrimagnetic GdFeCo alloys" Applied Physics Letters 109, 112403 (2016), https://doi.org/10.1063/1.4962812

[6] C. Gong, S. Li, H. Zhang, T. Su, M. Hu, H. Ma et al. "Study on synthesis and electrical properties of slab shape diamond crystals in FeNiMnCo-C-P system under HPHT" International Journal of Refractory Metals and Hard Materials 66, 116-121 (2017), https://doi.org/10.1016/j.ijrmhm.2017.03.003

[7] V. Nadolinny, A. Komarovskikh, Y. Palyanov, A. Sokol "EPR of synthetic diamond heavily doped with phosphorus" Physica Status Solidi (a) 212 (11), 2568-2571 (2015), https://doi.org/10.1002/pssa.201532166

[8] Y. Balasubramaniam, P. Pobedinskas. S.D. Janssens, S.D., G. Sakr, F. Jomard, S. Turner, Y.-G. Lu, W. Dexters, A. Soltani, J. Verbeeck, J. Barjon, M. Nesladek, K. Haenen, "Thick homoepitaxial (110)-oriented phosphorus-doped n-type diamond" Applied Physics Letters 109 (6), 062105 (2016), https://doi.org/10.1063/1.4960970

[9] M.-A. Pinault-Thaury, B. Berini, I. Stenger, E. Chikoidze, A. Lusson, F. Jomard et al. "High fraction of substitutional phosphorus in a (100) diamond epilayer with low surface roughness" Applied Physics Letters 100, 192109 (2012), http://dx.doi.org/10.1063/1.4712617

[10] M. Lesik, T. Plays, A. Tallaire, J. Achard, O. Brinza, L. William et al. "Preferential orientation of NV defects in CVD diamond films grown on (113)-oriented substrates" Diamond and Related Materials 56, 47-53 (2015), https://doi.org/10.1016/j.diamond.2015.05.003

[11] K.A. Snail, Z.P. Lu, R.Weimer, J. Heberlein, E. Pfender, L.M. Hanssen "Confirmation of $\{113\}$ facets on diamond grown by chemical vapor deposition" Journal of Crystal Growth 137, 676-679 (1994), https://doi.org/10.1016/j.diamond.2015.05.003

[12] F. Silva, J. Achard, X. Bonnin, O. Brinza, A. Michau, A. Secroun et al. "Single crystal CVD diamond growth strategy by the use of a 3D geometrical model: growth on (113) oriented substrates" Diamond and Related Materials 17 (7-10), 1067-1075 (2008), https://doi.org/10.1016/j.diamond.2008.01.006

[13] A. Tallaire, A. Valentin, V. Mille, L. William, M.-A. Pinault-Thaury, F. Jomard et al. "Growth of thick and heavily boron-doped (113)-oriented CVD diamond films" Diamond and Related Materials 66, 61-66 (2016), https://doi.org/10.1016/j.diamond.2016.03.020

[14] S. Koizumi, M. Kamo, Y. Sato, H. Ozaki, T. Inuzuka "Growth and characterization of phosphorous doped $\{111\}$ homoepitaxial diamond thin films" Applied Physics Letters 71 (8), 1065 (1997), https://doi.org/10.1063/1.119729

[15] S. Koizumi, T. Teraji, H. Kanda "Phosphorus-doped chemical vapor deposition of diamond" Diamond and Related Materials 9 (3-6), 935-940 (2000), https://doi.org/10.1016/S0925-9635(00)00217-X

[16] T. Kociniewski, M.-A. Pinault, J. Barjon, F. Jomard, J. Chevallier, C. Saguy "MOCVD doping technology for phosphorus incorporation in diamond: Influence of the growth 
temperature on the electrical properties" Diamond and Related Materials 16 (4-7), 815818 (2007), https://doi.org/10.1016/j.diamond.2006.12.032

[17] M.-A. Pinault-Thaury, T. Tillocher, D. Kobor, N. Habka, F. Jomard, J. Chevallier et al. "Phosphorus donor incorporation in ( 1000$)$ homoepitaxial diamond: Role of the lateral growth" Journal of Crystal Growth 335 (1), 31-36 (2011), https://doi.org/10.1016/j.jcrysgro.2011.06.007

[18] H. Kato, S. Yamasaki, H. Okushi "n-type doping of (001)-oriented single-crystalline diamond by phosphorus" Applied Physics Letters 86 (22), 222111 (2005), https://doi.org/10.1063/1.1944228

[19] M. Hasegawa, T. Teraji, S. Koizumi "Lattice location of phosphorus in n-type homoepitaxial diamond films grown by chemical-vapor deposition" Applied Physics Letters 79 (19), 3068 (2001), https://doi.org/10.1063/1.1417514

[20] H. Kato, T. Makino, S. Yamasaki, H. Okushi "n-type diamond growth by phosphorus doping on (0 0 1)-oriented surface" Journal of Physics D: Applied Physics 40 (20), 6189-6200 (2007), http://dx.doi.org/10.1088/0022-3727/40/20/S05

[21] M.-A. Pinault-Thaury, S. Temgoua, R. Gillet, H. Bensalah, I. Stenger, F. Jomard et al. "Phosphorus-doped (113) CVD diamond: A breakthrough towards bipolar diamond devices" Applied Physics Letters 114 (11), 112106, (2019), https://doi.org/10.1063/1.5079924

[22] T. Nakai, O. Maida, T. Ito "Characterization of phosphorus-doped homoepitaxial (100) diamond films grown using high-power-density MWPCVD method with a conventional quartz-tube chamber” Applied Surface Science 254 (9), 6281 (2008), https://doi.org/10.1016/j.apsusc.2008.02.167

[23] O. Maida, S. Tada, H. Nishio, T. Ito "Substrate temperature optimization for heavilyphosphorus-doped diamond films grown on vicinal (001) surfaces using high-powerdensity microwave-plasma chemical-vapor-deposition" Journal of Crystal Growth 424, 33-37 (2015), https://doi.org/10.1016/j.jcrysgro.2015.04.037

[24] N. Donato, N. Rouger, J. Pernot, G. Longobardi and F. Udrea, "Diamond power devices: state of the art, modelling, figures of merit and future perspective" Journal of Physics D 53, 093001 (2020), https://doi.org/10.1088/1361-6463/ab4eab

[25] T. Kociniewski, J. Barjon, M.-A. Pinault, F. Jomard, A. Lusson, D. Ballutaud et al. "ntype CVD diamond doped with phosphorus using the MOCVD technology for dopant incorporation" Physica Status Solidi (a) 203 (12), 3136-3141 (2006), https://doi.org/10.1002/pssa.200671113

[26] J. Barjon, P. Desfonds, M.-A. Pinault, T. Kociniewski, F. Jomard, J. Chevallier 'Determination of the phosphorus content in diamond using cathodoluminescence spectroscopy' Journal of Applied Physics 101 (11), 113701 (2007); https://doi.org/10.1063/1.2735408

[27] N. Temahuki, R. Gillet, V. Sallet, F. Jomard, E. Chikoidze, Y. Dumont et al. "New Process for Electrical Contacts on (100) N-type Diamond" Physica Status Solidi (a) 214 (11), 1700466 (2017), https://doi.org/10.1002/pssa.201700466

[28] J. Barjon and K. Haenen "Optical spectroscopy of doped diamond layers" in Power Electronics Device Applications of Diamond Semiconductors, edited by S. Koizumi, H. Umezawa, J. Pernot, and M. Suzuki (Woodhead Publishing, 2018), Part 2.4, 154-173

[29] J. Barjon "Luminescence Spectroscopy of Bound Excitons in Diamond" Physica Status Solidi (a) 214 (11), 1700402 (2017), https://doi.org/10.1002/pssa.201700402

[30] N. Teofilov, R. Sauer, K. Thonke, S. Koizumi "Bound exciton luminescence related to phosphorus donors in CVD diamond" Physica B 340-342, 99-105 (2003), https://doi.org/10.1016/j.physb.2003.09.011 
[31] I. Stenger, M.-A. Pinault-Thaury, A. Lusson, T. Kociniewski, F. Jomard, J. Chevallier et al. "Quantitative analysis of electronic absorption of phosphorus donors in diamond" $\begin{array}{lllll}\text { Diamond and } & \text { Related }\end{array}$ https://doi.org/10.1016/j.diamond.2017.01.012

[32] Mott N. F. and DAVIS E. A., Electronic Processes in Noncrystalline Materials, 2nd edition (Oxford University Press) 1979

[33] I. Stenger, M.-A. Pinault-Thaury, T. Kociniewski, A. Lusson, E. Chikoidze, F. Jomard et al. "Impurity-to-band activation energy in phosphorus doped diamond" Journal of Applied Physics 114 (7), 073711 (2013), https://doi.org/10.1063/1.4818946

[34] S. Koizumi, M. Suzuki "n-Type doping of diamond" Physica Status Solidi (a) 203 (13), 3358-3366 (2006), https://doi.org/10.1002/pssa.200671407

[35] H. Kato, T. Makino, S. Yamasaki, H. Okushi "n-Type Diamond Gowth by Phosphorus Doping" Material Research Society Proceedings 1039 (Symposium P - Diamond $\begin{array}{lllll}\text { Electronics-Fundamentals to Applications } & \text { II), } & 05 & \text { (2007), }\end{array}$ https://doi.org/10.1557/PROC-1039-P05-01

[36] H. Kato, J. Barjon, N. Habka, T. Matsumoto, D. Takeuchi, H. Okushi e al. "Energy level of compensator states in (001) phosphorus-doped diamond" Diamond and Related Materials 20 (7), 1016-1019 (2011), https://doi.org/10.1016/j.diamond.2011.05.021

[37] R. Jones, J.E. Lowther, J. Goss, "Limitations to n-type doping in diamond: The phosphorus-vacancy complex" Applied Physics Letters (1996), 69 (17), 2489 (1996), https://doi.org/10.1063/1.117715

[38] J. Pernot and S. Koizumi, "Electron mobility in phosphorus doped $\{111\}$ homoepitaxial diamond", Applied Physics Letters 93, $05105 \quad$ (2008), https://doi.org/10.1063/1.2969066

[39] J. Pernot, C. Tavares, E. Gheeraert, E. Bustarret, M. Katagiri, S. Koizumi, "Hall electron mobility in diamond" Applied Physics Letters 89 (12), 122111 (2006), https://doi.org/10.1063/1.2355454 